\title{
On gamma inverse Weibull distribution
}

\author{
Salman Abbas ${ }^{\text {** }}$, Mahjabeen Hameed ${ }^{2}$, Selen Cakmakyapan ${ }^{3}$ and Sarfraz Nawaz Malik ${ }^{4}$ \\ ${ }^{1}$ Department of Statistics, COMSATS University Islamabad, Lahore Campus, Pakistan. \\ ${ }^{2}$ Department of Statistics and Computer Science, University of Veterinary and Animal Sciences, Pakistan. \\ ${ }^{3}$ Department of Statistics, Hacettepe University, Ankara, Turkey. \\ ${ }^{4}$ Department of Mathematics, COMSATS University Islamabad, Wah Campus, Pakistan.
}

Submitted: 11 September 2018; Revised: 26 April 2019; Accepted: 23 August 2019

\begin{abstract}
The inverse Weibull distribution which was first introduced to investigate the decay of mechanical components is widely used in the field of biology, especially in the areas of survival and reliability analysis. In this paper, a generalisation of inverse Weibull distribution is presented using a new class of gamma distributions. For the proposed model, important mathematical properties including quantile function, noncentral moment, central moment, moment generating function, characteristics function, and general coefficient are discussed. The shapes of the probability density function and risk function were obtained and the graphical demonstrations of them were provided. To estimate model parameters of the proposed method, maximum likelihood was used. An illustrative simulation study was conducted to observe how the maximum likelihood estimators behave for different sample sizes and for different values of parameters. Two real data sets from the field of biomedical sciences were used to demonstrate the practical applications of the proposed model in real life phenomena. Results were obtained from the data sets, using the gamma inverse Weibull distribution and some known existing distributions and compared. The comparative analysis justified that the proposed model significantly fits with the survival data compared to other distributions.
\end{abstract}

Keywords: Gamma family of distribution, general coefficient, inverse Weibull distribution, maximum likelihood.

\section{INTRODUCTION}

The inverse Weibull (IW) distribution is widely used in reliability analysis. Keller and Kanath (1982) first introduced IW distribution to investigate the decay of mechanical components. Nelson (1982) used the IW distribution for modelling of survival data to determine the time to breakdown insulating fluid and subject to the action of constant tension. Calabria and Pulcini (1990) discussed the estimation of parameter for IW distribution using method of maximum likelihood and least square. Mahmoud et al. (2003) described the recurrence relations for the single and product moments of order statistics for both non-truncated and truncated IW distribution. Aleem and Pasha (2003) defined the single, product, and ratio moments of order statistics of IW distribution. de Gusmão et al. (2011) introduced the generalised IW distribution and Hanook et al. (2009) developed the beta IW distribution. Using distribution function (cdf) of Kumaraswamy family of distribution, Shahbaz et al. (2012) computed the Kumaraswamy IW distribution. For lifetime modelling, IW distribution was generalised by Shuaib et al. (2016). Abbas et al. (2017) developed the Topp Leone IW distribution.

Generalisation of classical models is a widely practiced method in statistical analysis. To handle real data most of the time existing classical distributions are modified by adding one or more parameters.

In this article, we propose a new generalisation of IW distribution using a new class of gamma distribution introduced by Brito et al. (2017). The proposed model is named as gamma inverse Weibull distribution (GIW). The new model is more adaptable and flexible for the modelling of survival datasets.

\footnotetext{
* Corresponding author (salmanabbas@cuilahore.edu.pk; (iD https://orcid.org/0000-0003-4724-4603)
} 
The cdf of gamma $\left[\frac{(1-G)}{G}\right]$ class of distribution is given as follows;

$$
\begin{gathered}
F(y)=1-\int_{0}^{\frac{1-G(y)}{G(y)}} \frac{b^{a}}{\Gamma(a)} t^{a-1} e^{-b t} d t=Q\left[a, b \frac{1-G(y)}{G(y)}\right], \\
\text { a,b }>0
\end{gathered}
$$

where $Q(\alpha, t)=\frac{\Gamma(\alpha, t)}{\Gamma(\alpha)}$ is regular incomplete gamma function, $\quad \Gamma(\alpha, t)=\int_{t}^{\infty} t^{\alpha-1} e^{-y} d y, z \geq 0, \quad$ is the incomplete gamma function, and $\Gamma(a)$ is gamma function. By differentiating equation (1), the probability density function (pdf) of the proposed class is given as follows;

$$
\begin{gathered}
f(y)=\frac{g(y)}{G^{2}(x)} \frac{b^{a}}{\Gamma(a)}\left[\frac{1-G(y)}{G(y)}\right]^{a-1} \exp \left[-b\left[\frac{1-G(y)}{G(y)}\right]\right], \\
\mathrm{a}, \mathrm{b}>0
\end{gathered}
$$

where $g(y)=\frac{d(G(y))}{d y}$

\section{GAMMA INVERSE WEIBULL DISTRIBUTION}

In this section, we derive the cdf and pdf of gamma inverse Weibull distribution (GIW). Let $Y$ follow the IW distribution. Then the cdf of one parameter IW distribution is given as,

$$
G(y)=e^{-y^{-\delta}}, \mathrm{y}>0, \delta>0,
$$

and the corresponding pdf is as,

$$
g(y)=\delta y^{-(\delta+1)} e^{-y^{-\delta}}, \mathrm{y}>0, \delta>0,
$$

Inserting equation (3) in equation (1), we have the cdf of GIW distribution as follows;

$$
F(y)=1-\int_{0}^{\frac{1-e^{-y^{-\delta}}}{e^{-y^{-\delta}}}} \frac{b^{a}}{\Gamma(a)} t^{a-1} e^{-b t} d t=Q\left[a, b \frac{1-e^{-y^{-\delta}}}{e^{-y^{-\delta}}}\right]
$$

$\mathrm{y}>0, \mathrm{a}, \mathrm{b}, \delta>0$,

The corresponding density of the GIW distribution is obtained by taking derivative of (5) and is given as,

$$
f(y)=\frac{\delta y^{-(\delta+1)} e^{-y^{-\delta}}}{\left(e^{-y^{-\delta}}\right)^{2}} \frac{b^{a}}{\Gamma(a)}\left[\frac{1-e^{-y^{-\delta}}}{e^{-y^{-\delta}}}\right]^{a-1} \exp \left[-b\left[\frac{1-e^{-y^{-\delta}}}{e^{-y^{-\delta}}}\right]\right]
$$

$\mathrm{y}>0, \mathrm{a}, \mathrm{b}, \delta>0$,
For $\delta=1$, the proposed model reduces to gamma inverse exponential distribution. The new model converts into gamma inverse Rayleigh distribution for $\delta=2$.

The cfd and the pdf of the GIW distribution is expressed in form of exponentiated G-distributions as,

$$
F(y)=\sum_{i, j=0}^{\infty} \frac{(-1)^{i+j} b^{a+i}}{i !(j-a-i) \Gamma(a)}\left(\begin{array}{c}
i+a-1 \\
j
\end{array}\right)\left(e^{-y^{-\delta}}\right)^{j-a+i},
$$

and

$f(y)=\sum_{i, j=0}^{\infty} \frac{(-1)^{i+j} b^{a+i}}{i ! \Gamma(a)}\left(\begin{array}{c}i+a-1 \\ j\end{array}\right) \delta y^{-(\delta+1)}\left(e^{-y^{-\delta}}\right)^{j-a-i}$.

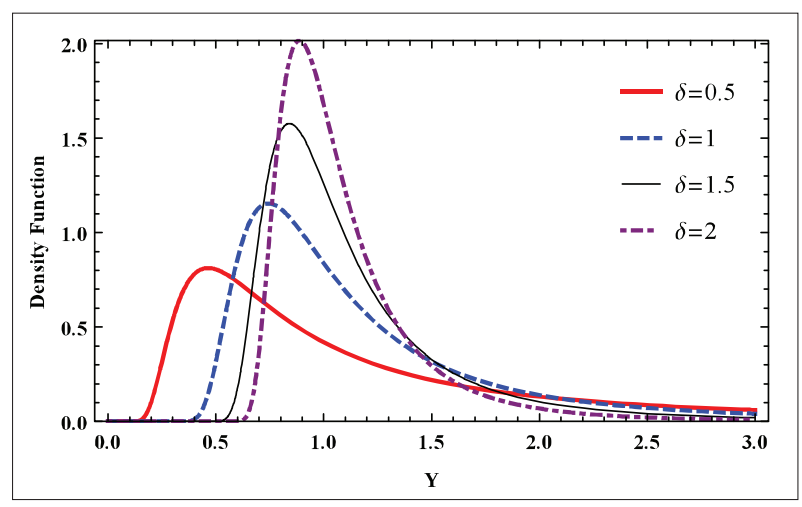

Figure 1: The pdf of GIW distribution for $a=2, b=1$ and various values of $\delta$

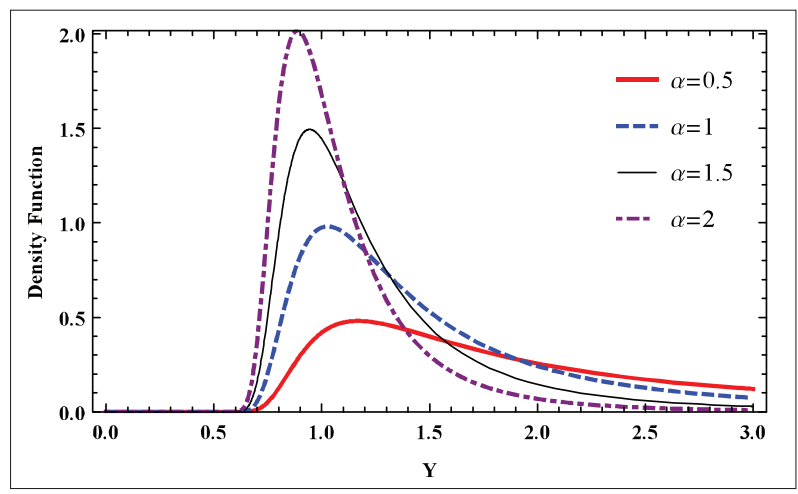

Figure 2: The pdf of GIW distribution for $\delta=2, b=1$ and various values of $a$ 


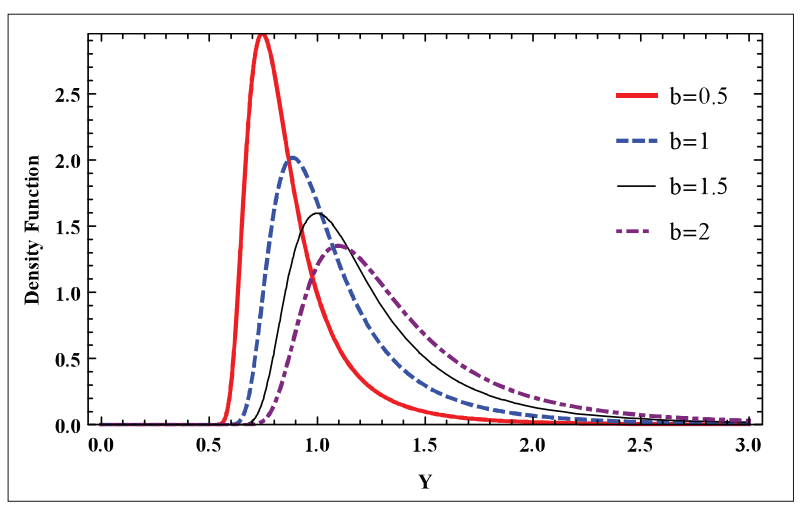

Figure 3: The pdf of $G I W$ distribution for $\delta=2, a=2$ and various values of $b$

Figure 1 displays the pdf of GIW distribution for $\mathrm{a}=2$, $\mathrm{b}=1$ with different values of $\delta$. It is noted that with the increase in $\delta$ value the distribution remains positively skewed but its peak increases.

Figure 2 displays the pdf of GIW distribution for $\delta=2, \mathrm{~b}=1$ with different values of $a$. It is noted that with the increase in $a$ value the distribution again remains positively skewed and its peak increases, but there is no expressing change in the location and shape of the distribution.

Figure 3 displays the pdf of GIW distribution for $\delta=2, \mathrm{a}=2$ with different values of $b$. It is noted that as we increase the value of $b$ the peak decreases. For $b<1$ the function initially shows a rapid increase and then slowly decreases as we increase the $\mathrm{y}$ - value, but for $b>1$ the function initially shows a slow increase and the curve becomes flattened as $y$ increases.

Figure 4 displays the plot of pdf of GIW distribution for various values of $\delta$ and $b$ when $a=2$. These plots clearly indicate that the GIW distribution is unimodel.

\section{PROPERTIES OF GAMMA INVERSE WEIBULL DISTRIBUTION}

In this section, we derive and discuss some useful mathematical characteristics of the GIW distribution.
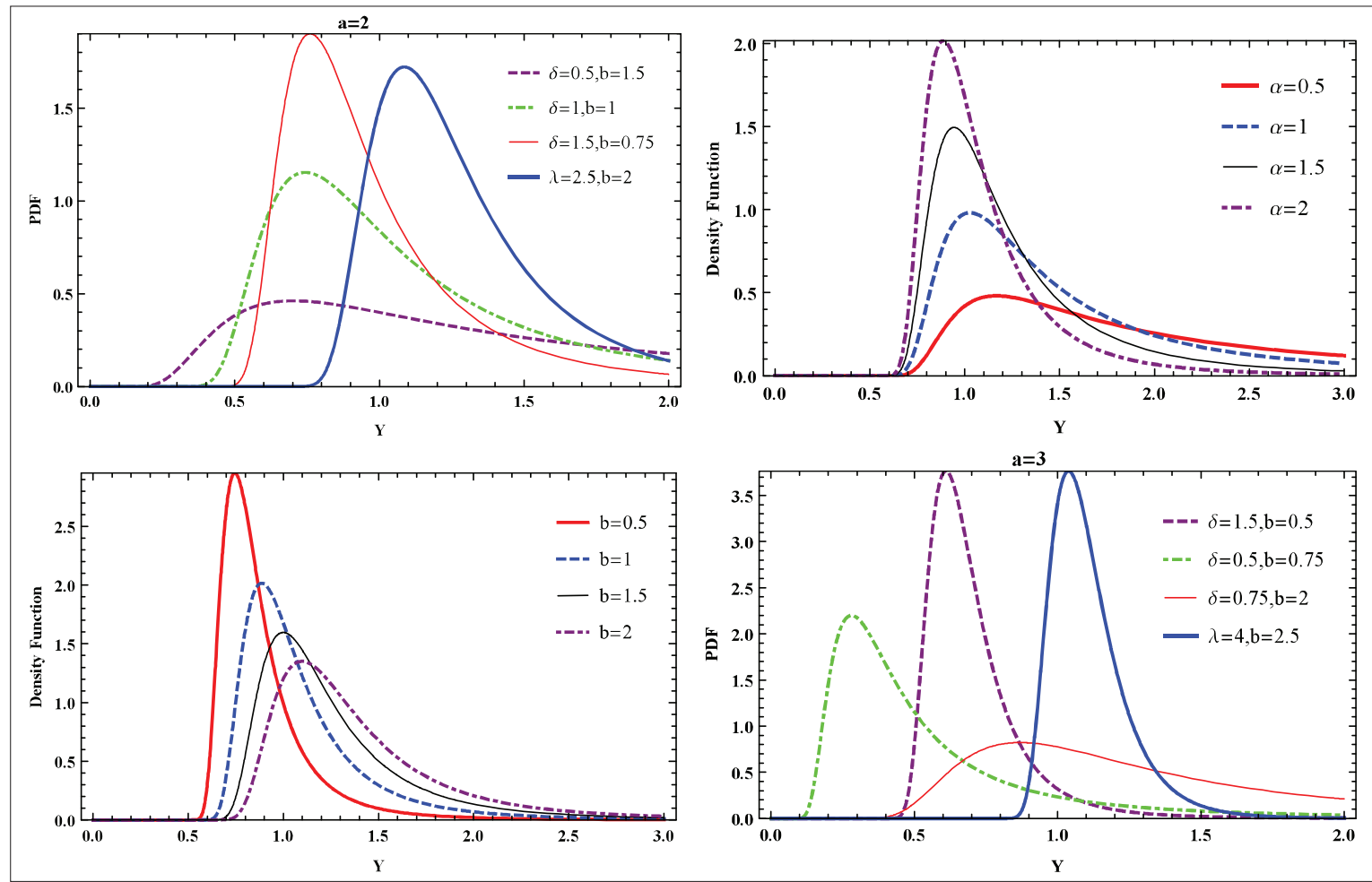

Figure 4: The graphs for density function of GIW distribution for various values of $a, b$, and $\delta$ 


\section{Moments, moment generating and characteristics function}

Moment is an interesting feature in distribution theory. It is used to define several important characteristics of the probability model including tendency, dispersion, skewness and kurtosis. The general expression for raw moment is given as,

$\mu_{p}=\int_{0}^{\infty} y^{p} f(y) d y$.

Using equation (8) and performing some calculations, the $p-t h$ moment of the GIW distribution is obtained as,

$\mu_{p}=\sum_{i, j=0}^{\infty} \frac{(-1)^{i+j} b^{a+i}}{i ! \Gamma(a)}\left(\begin{array}{c}i+a-1 \\ j\end{array}\right)\left(\frac{1}{j-a-i}\right)^{1-\frac{p}{\delta}} \Gamma\left(1-\frac{p}{\delta}\right)$.

For the above expression, $\delta>p$

The moment generating function of the proposed model is also obtained using equation (8). The general expression if given as,

$M_{y}(t)=\int_{0}^{\infty} e^{t y} \sum_{i, j=0}^{\infty} \frac{(-1)^{i+j} b^{a+i}}{i ! \Gamma(a)}\left(\begin{array}{c}i+a-1 \\ j\end{array}\right) \delta y^{-(\delta+1)}\left(e^{-y^{-\delta}}\right)^{j-a-i} d y$,

where

$e^{t y}=\sum_{m=0}^{\infty} \frac{t^{m}}{m !}$

After some simplification, the formulation is derived and presented as,

$$
\begin{aligned}
M_{y}(t)= & \sum_{i, m, j=0}^{\infty} \frac{(-1)^{i+j} b^{a+i} t^{m}}{i ! \Gamma(a)}\left(\begin{array}{c}
i+a-1 \\
j
\end{array}\right)\left(\frac{1}{j-a-i}\right)^{1-\frac{m}{\delta}} \Gamma\left(1-\frac{m}{\delta}\right), \\
& t>0 .
\end{aligned}
$$

Similarly, the characteristic function of the GIW distribution is provided as,

$\phi_{y}(t)=\sum_{m, j=0}^{\infty} \frac{(-1)^{i+j} b^{a+i} t^{m} i^{m}}{i ! \Gamma(a)}\left(\begin{array}{c}i+a-1 \\ j\end{array}\right)\left(\frac{1}{j-a-i}\right)^{1-\frac{m}{\delta}} \Gamma\left(1-\frac{m}{\delta}\right)$.

\section{Central moments and general coefficient}

Here, we derive and discuss the moment about origin and general coefficient. The expression of moment about origin of the gamma class of distributions is given as,

$\mu_{m}^{\prime}=\sum_{r=0}^{m} \sum_{i, j=0}^{\infty} \frac{(-1)^{i+j+r} b^{a+i} \mu^{r}}{i ! \Gamma(a)}\left(\begin{array}{c}m \\ r\end{array}\right)\left(\begin{array}{c}i+a-1 \\ j\end{array}\right) \psi_{m-r, 0, j-a-i-1}$, where $\psi_{m-r, 0, j-a-i-1}=\int_{-\infty}^{\infty} x^{m-r} g(y) G(y)^{j-a-i-1} d y$ and $\mu^{r}$ is the non-central moment of the distribution. Moment about origin of the GIW distribution is conferred as,

$$
\begin{aligned}
\mu_{m}^{\prime}= & \sum_{r=0}^{m} \sum_{i, j=0}^{\infty} \frac{(-1)^{i+j+r} b^{a+i} \mu^{r}}{i ! \Gamma(a)}\left(\begin{array}{c}
m \\
r
\end{array}\right)\left(\begin{array}{c}
i+a-1 \\
j
\end{array}\right)\left(\frac{1}{j-a-i}\right)^{1-\frac{m}{\delta}} \\
& \Gamma\left(1-\frac{m}{\delta}\right) .
\end{aligned}
$$

The variance of the GIW distribution is computed by replacing $\mathrm{m}=2$ in the above expression and provided as,

$$
\begin{aligned}
\sigma=\mu_{2}^{\prime}= & \sum_{r=0}^{2} \sum_{i, j=0}^{\infty} \frac{(-1)^{i+j+r} b^{a+i} \mu^{r}}{i ! \Gamma(a)}\left(\begin{array}{l}
2 \\
r
\end{array}\right)\left(\begin{array}{c}
i+a-1 \\
j
\end{array}\right)\left(\frac{1}{j-a-i}\right)^{1-\frac{2}{\delta}} \\
& \Gamma\left(1-\frac{2}{\delta}\right) .
\end{aligned}
$$

General coefficient is a new abstraction for measuring the skewness and kurtosis of the distribution. So, the general coefficient of the proposed model is established and demonstrated as,

$$
C_{g}(m)=\frac{\sum_{r=0}^{m} \sum_{i, j=0}^{\infty} \frac{(-1)^{i+j+r} b^{a+i} \mu^{r}}{i ! \Gamma(a)}\left(\begin{array}{c}
m \\
r
\end{array}\right)\left(\begin{array}{c}
i+a-1 \\
j
\end{array}\right)\left(\frac{1}{j-a-i}\right)^{1-\frac{m}{\delta}} \Gamma\left(1-\frac{m}{\delta}\right)}{\left[\sum_{r=0}^{2} \sum_{i, j=0}^{\infty} \frac{(-1)^{i+j+r} b^{a+i} \mu^{r}}{i ! \Gamma(a)}\left(\begin{array}{l}
2 \\
r
\end{array}\right)\left(\begin{array}{c}
i+a-1 \\
j
\end{array}\right)\left(\frac{1}{j-a-i}\right)^{1-\frac{2}{\delta}} \Gamma\left(1-\frac{2}{\delta}\right) .\right]^{\frac{m}{2}}} .
$$

Skewness and kurtosis of the GIW distribution can be obtained by inserting $\mathrm{m}=3$ and $\mathrm{m}=4$.

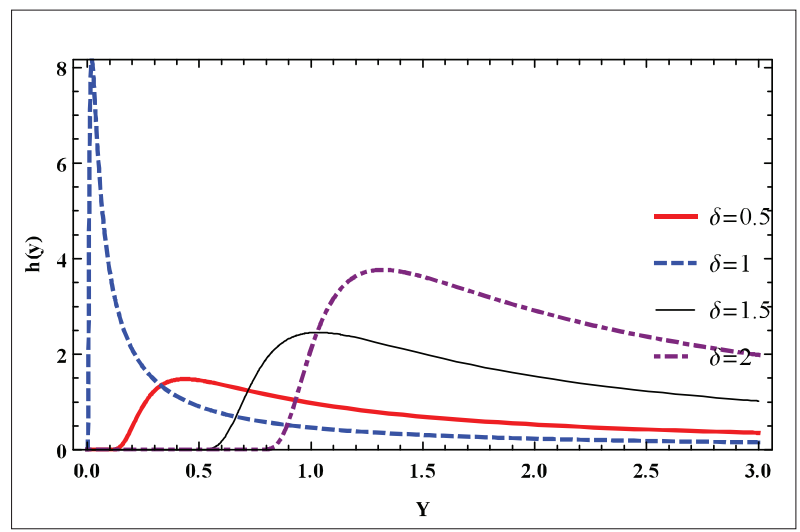

Figure 5: The Risk Function of GIW distribution for $a=1, b=1$ and various values of $\delta$ 


\section{Quantile and risk function}

The $u^{\text {th }}$ quantile function of GIW distribution is derived and given as,

$$
F_{y}^{-1}(u)=-\left[\log \left[\frac{b}{\left[Q^{-1}(a, u)+b\right]}\right]\right]^{-\frac{1}{\delta}},
$$

where $Q^{-1}(a, u)$ is the inverse function of regular incomplete gamma function.

The risk function of any distribution is obtained by using the following method;

$R(y)=\frac{f(y)}{1-F(y)}$.

Using equations (5) and (6), the risk function of GIW distribution is obtained as,

$$
R(y)=\frac{\frac{\delta y^{-(\delta+1)} e^{-y^{-\delta}}}{\left(e^{-y^{-\delta}}\right)^{2}} \frac{b^{a}}{\Gamma(a)}\left[\frac{1-e^{-y^{-\delta}}}{e^{-y^{-\delta}}}\right]^{a-1} \exp \left[-b\left[\frac{1-e^{-y^{-\delta}}}{e^{-y^{-\delta}}}\right]\right]}{1-Q\left[a, b \frac{1-e^{-y^{-\delta}}}{e^{-y^{-\delta}}}\right]},
$$

Figure 5 reveals that the hazard rate is upward-down shape. The survival function of the proposed distribution is given as,

$$
S(y)=1-Q\left[a, b \frac{1-e^{-y^{-\delta}}}{e^{-y^{-\delta}}}\right]
$$

\section{Mode}

For the mode, the density of GIW distribution given in

equation (6) is considered. The expression of mode is obtained by solving $\frac{\partial f(y)}{\partial y}$ and given as,

$f^{\prime}(y)=-\frac{\delta b^{a} y^{-2(\delta+1)}(1-w)^{a-2} e^{-b w+b-\delta+2 y^{-\delta}-y}\left(\delta\left(-(a+b+1) w+b w^{2}+2\right)+(1-w) y^{\delta}(\delta+y+1)\right)}{\Gamma(a)}$

Equating $\frac{\partial f(y)}{\partial y}=0$, we have

$$
-\frac{\delta b^{a} y^{-2(\delta+1)}(1-w)^{a-2} e^{-b w+b-\delta+2 y^{-\delta}-y}\left(\delta\left(-(a+b+1) w+b w^{2}+2\right)+(1-w) y^{\delta}(\delta+y+1)\right)}{\Gamma(a)}=0,
$$

where $w=e^{y^{-\delta}}$. Solving equation (11) iteratively, the maxima can be obtained.

\section{ESTIMATION}

In this section, the maximum likelihood method is adapted for estimation of the model parameters. The estimation is based on complete sample. Let $y_{1}, \ldots \ldots ., y_{n}$ be the random sample of size $n$ drawn from the density [equation (6)] of GIW distribution given as,

$$
f(y)=\frac{\delta y^{-(\delta+1)} e^{-y^{-\delta}}}{\left(e^{-y^{-\delta}}\right)^{2}} \frac{b^{a}}{\Gamma(a)}\left[\frac{1-e^{-y^{-\delta}}}{e^{-y^{-\delta}}}\right]^{a-1} \exp \left[-b\left[\frac{1-e^{-y^{-\delta}}}{e^{-y^{-\delta}}}\right]\right]
$$

The likelihood function of the above density is given as,

$$
\begin{aligned}
L(y, a, b, \delta)= & \prod_{i=0}^{n}\left[\frac{\delta y^{-(\delta+1)} e^{-y^{-\delta}}}{\left(e^{-y^{-\delta}}\right)^{2}} \frac{b^{a}}{\Gamma(a)}\left[\frac{1-e^{-y^{-\delta}}}{e^{-y^{-\delta}}}\right]^{a-1}\right. \\
& \left.\exp \left[-b\left[\frac{1-e^{-y^{-\delta}}}{e^{-y^{-\delta}}}\right]\right]\right],
\end{aligned}
$$

where $y=\left[y_{1}, y_{2}, \ldots . ., y_{n}\right]^{\prime}$. The log-likelihood function of density [equation (6)] is given as follows;

$$
\begin{aligned}
L(y ; a, b, \delta)= & n \log \left(\frac{b^{a}}{\Gamma(a)}\right)+\sum_{i=0}^{n} \log \left(\frac{\delta y_{i}^{-(\delta+1)} e^{-y_{i}^{-\delta}}}{\left(e^{-y_{i}^{-\delta}}\right)^{2}}\right)+ \\
& (a-1) \sum_{i=0}^{n} \log \left(\frac{1-e^{-y_{i}^{-\delta}}}{e^{-y_{i}^{-\delta}}}\right)-b \sum_{i=0}^{n} \frac{1-e^{-y_{i}^{-\delta}}}{e^{-y_{i}^{-\delta}}}
\end{aligned}
$$

The partial derivatives of log-likelihood function in equation (12) w:r:t parameters $a, b, \delta$ are given as follows:

$$
\begin{aligned}
& \frac{\partial(L)}{\partial a}=n \log b-n \psi(a)+\sum_{i=0}^{n} \log \left(\frac{1-e^{-y^{-\delta}}}{e^{-y^{-\delta}}}\right) \\
& \frac{\partial(L)}{\partial b}=\frac{n a}{b}-\sum_{i=0}^{n} \frac{1-e^{-y^{-\delta}}}{e^{-y^{-\delta}}}
\end{aligned}
$$




$$
\begin{aligned}
\frac{\partial(L)}{\partial \delta}= & \sum_{i=0}^{n} \frac{e^{-y^{-\delta}} y^{\delta+1}\left(e^{y^{-\delta}} y^{-\delta-1}+\delta\left(-e^{y^{-\delta}}\right) y^{-2 \delta-1} \log (y)-\delta e^{y^{-\delta}} y^{-\delta-1} \log (y)\right)}{\delta} \\
& +(a-1) \sum_{i=0}^{n} \frac{e^{-y^{-\delta}}\left(-e^{y^{-\delta}}\left(1-e^{-y^{-\delta}}\right) y^{-\delta} \log (y)+y^{-\delta} \log (y)\right)}{1-e^{-y^{-\delta}}}+b \sum_{i=0}^{n}\left(e^{y^{-\delta}}\left(1-e^{-y^{-\delta}}\right) y^{-\delta} \log (y)+y^{-\delta} \log (y)\right),
\end{aligned}
$$

where $\psi$ is the diagamma function. The maximum likelihood estimates $\hat{a}, \hat{b}$, and $\hat{\delta}$ of the parameters $a, b$ and $\delta$ are defined by equating (13), (14), and (15), equal to zero and solved them iteratively. The minus expectation of the second partial derivatives of equations (13),(14), and (15) exhibit the result of Fisher information matrix $I(\delta, a, b)$. The variance and covariance are extracted from the inverse of Fisher' information matrix.

Table 1: $\quad$ Simulation results for Set 1, Set 2, and Set 3

\begin{tabular}{lccccccccc}
\hline $\mathrm{n}$ & $\delta=0.1$ & $\mathrm{a}=0.2$ & $\mathrm{~b}=0.3$ & $\delta=0.2$ & $\mathrm{a}=0.3$ & $\mathrm{~b}=0.1$ & $\delta=0.3$ & $\mathrm{a}=0.2$ & $\mathrm{~b}=0.1$ \\
\hline \multirow{2}{*}{30} & 0.1375 & 0.1627 & 0.2857 & 0.2288 & 0.2988 & 0.1312 & 0.3491 & 0.1654 & 0.1626 \\
& $(0.0644)$ & $(0.3261)$ & $(0.1952)$ & $(0.068)$ & $(0.3579)$ & $(0.4179)$ & $(0.1236)$ & $(0.5645)$ & $(0.4196)$ \\
\multirow{2}{*}{100} & 0.1096 & 0.193 & 0.2963 & 0.2094 & 0.2979 & 0.1008 & 0.3139 & 0.1487 & 0.1381 \\
& $(0.0235)$ & $(0.0463)$ & $(0.0974)$ & $(0.0306)$ & $(0.0633)$ & $(0.0515)$ & $(0.0509)$ & $(0.9597)$ & $(0.7238)$ \\
500 & 0.1005 & 0.2022 & 0.3067 & 0.2014 & 0.3007 & 0.1011 & 0.3003 & 0.1931 & 0.1115 \\
& $(0.0083)$ & $(0.0209)$ & $(0.0469)$ & $(0.0116)$ & $(0.0272)$ & $(0.0229)$ & $(0.0194)$ & $(0.3159)$ & $(0.2201)$ \\
1000 & 0.1003 & 0.2014 & 0.3034 & 0.2008 & 0.3003 & 0.1005 & 0.2993 & 0.189 & 0.1081 \\
& $(0.0055)$ & $(0.0135)$ & $(0.0314)$ & $(0.0088)$ & $(0.0209)$ & $(0.0174)$ & $(0.0131)$ & $(0.4432)$ & $(0.1552)$ \\
\hline
\end{tabular}

\section{SIMULATION STUDY}

We organised an illustrative simulation study to see how the maximum likelihood estimators behave for different sample sizes and for different values of parameters. The sample sizes, namely, $\mathrm{n}=30,100,500$ and 1000 have three different sets of parameter values ( Set 1: $\delta=0.1$, $a=0.2, b=0.3$; Set 2: $\delta=0.2, a=0.3, b=0.1$; Set 3: $\delta=0.3, a=0.2, b=0.1$ ). In each case, the maximum likelihood estimator of the unknown parameters is computed by maximising the log-likelihood function. The average estimates and mean squared errors (in parenthesis) are computed over 1000 replications and the results are reported in Table 1. In all the cases, the performance of the maximum likelihood estimates are quite satisfactory. As sample size increases, the average estimates and the mean squared error decrease for all

Table 2: Tubercle bacilli data

121522242432323334383843444852535454555657585859 606060606162636565676870707273757676818384858791 95969899109110121127129131143146146175175211233258 258263297341341376 the parameters, as expected. It verifies the consistency properties of the MLEs.

Table 3: Fitted distributions and their abbreviations

\begin{tabular}{lc}
\hline Distributions & Abbreviation \\
\hline Log-Lindley-Weibull & LLW \\
Beta Weibull & BW \\
Kumaraswamy- Weibull & $\mathrm{KW}$ \\
McDonald- Weibull & $\mathrm{McW}$ \\
Marshall-Olkin-Weibull & $\mathrm{MOW}$ \\
Weibull- Weibull & $\mathrm{WW}$ \\
Lomax- Weibull & LoW \\
Lindley- Weibull & LiW \\
Topp-Leone-Weibull & $\mathrm{TW}$ \\
Gompertz-Weibull & $\mathrm{GW}$ \\
Odd Lindley-Weibull & OLW \\
Weighted Exponential & WE \\
Two-parameter Weighted Exponential & TWE \\
Extended Weighted Exponential & EWE \\
Extended Exponential & EE \\
Lindley & $\mathrm{L}$ \\
Exponential & $\mathrm{E}$ \\
Weibull & $\mathrm{W}$
\end{tabular}




\section{APPLICATION}

In this section, we provide the applicability of the GIW model in real life phenomena. For this purpose, we choose two datasets from the field of biomedical science. We obtained maximum likelihood estimates (MLE's), AIC, CAIC, and BIC statistics of GIW model and well known existing distributions mentioned in Table 3.
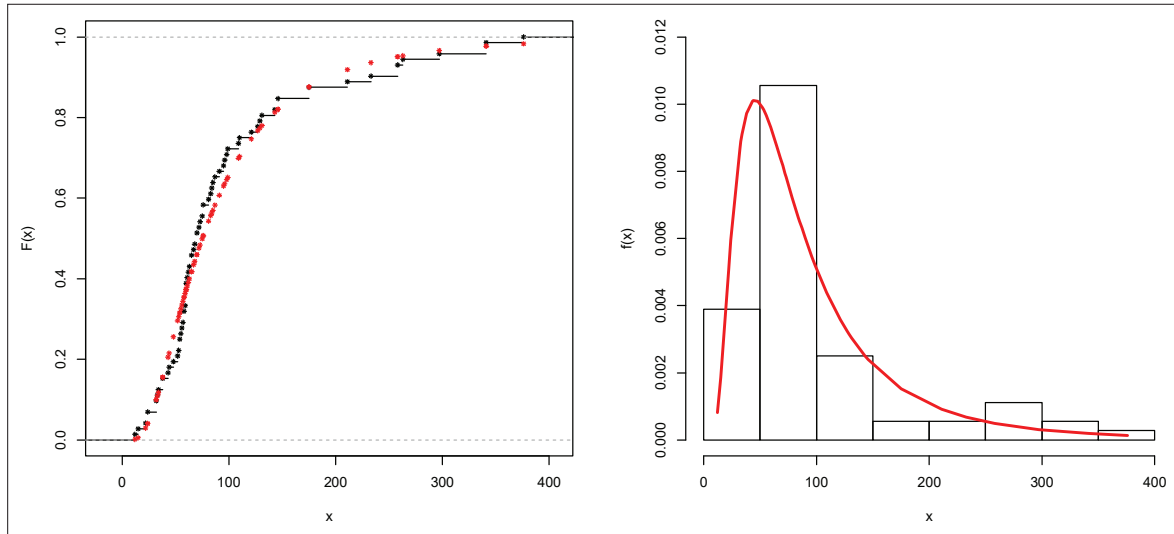

Figure 6: The histogram and the fitted empirical and GIW distribution for tubercle bacilli data

Table 4: MLEs, AIC, CAIC and BIC statistics of the fitted distributions of dataset

\begin{tabular}{lclll}
\hline Distribution & Parameters & AIC & CAIC & BIC \\
\hline GIW & $0.1757211,42.4673679,70.5647344$ & 786.7857 & 787.1386 & 793.6157 \\
EE & $10.1738,0.0200$ & 792.6086 & 792.7825 & 797.1619 \\
WE & $1.6312,0.0138$ & 791.1381 & 791.312 & 795.6914 \\
TWE & $2.8013,2.8013,0.0142$ & 789.0153 & 789.3682 & 795.8453 \\
EWE & $3.9035,3.0313,0.0141$ & 788.7657 & 789.1186 & 795.5957 \\
L & 0.019841 & 789.04 & 791.04 & 791.1 \\
E & 0.010018 & 806.88 & 808.88 & 808.94 \\
LLW & $0.230493,0.547514,14.024860,0.047958$ & 787.7472 & 788.3442 & 796.8539 \\
TW & $0.49220,0.37141,6.00952,4.46868$ & 788.1929 & 788.79 & 797.2996 \\
GW & $0.0030390,0.8408393,2.4757193,6.9645592$ & 806.7376 & 807.3346 & 815.8442 \\
LoW & $0.0030609,2.0150751,1.2104142,23.8855555$ & 791.3026 & 791.8997 & 800.4093 \\
LiW & $1.187295,0.988639,0.017651$ & 794.9608 & 795.3138 & 801.7908 \\
OLW & $2.6443973,0.1650443,0.0068032$ & 802.4631 & 802.5624 & 809.0394 \\
WW & $0.177315,0.535990,9.582126,1.527191$ & 788.3174 & 788.9145 & 797.4241 \\
MOW & $0.563061,0.473979,80.404218$ & 798.0679 & 798.4209 & 804.8979 \\
McW & $0.25188,0.63229,12.93719,0.53561,1.44729$ & 790.0641 & 790.9732 & 801.4474 \\
KW & $0.951877,0.326516,49.594511,1.518053$ & 788.2858 & 788.8829 & 797.3925 \\
BW & $0.23649,0.64716,12.15966,0.51710$ & 788.0632 & 788.6602 & 797.1698 \\
W & $0.0028431,1.2587947$ & 799.6583 & 799.8322 & 804.2116 \\
\hline
\end{tabular}

Table 5: Relief times data

$1.1,1.4,1.3,1.7,1.9,1.8,1.6,2.2,1.7,2.7,4.1,1.8,1.5,1.2,1.4,3,1.7,2.3,1.6,2$ 
First, we consider the tubercle bacili dataset. Bjerkedal (1960) described that the data is comprising values of survival times of 72 Guinea pigs injected with virulent tubercle bacilli. Table 1 gives observed values of the survival times of Guinea pigs in days. This dataset is already studied and fitted with different models by Lazhar et al. (2017), Shanker et al. (2015), and Mahdavi and Jabbari (2017). Then the comparison is carried out by considering a few other competitive models. The values of the dataset are provided in Table 2. Table 3 gives the fitted distributions and their abbreviations. The Table 4 reveals that the GIW distribution yields the lowest values of these statistics and then explains the suitable fit to the datasets. The results suggest that the GIW distribution performs better than the other fitted distributions. Visual comparison of the histogram of the data with GIW distribution is given in Figure 6. The plot of empirical distribution function and GIW distribution function are also displayed in Figure 6.

Table 6: Fitted distrubutions and their abbreviations

\begin{tabular}{lc}
\hline Distribution & Abbreviation \\
\hline The odd Frechet inverse Weibull & OFIW \\
New modified Weibull & NMW \\
Generalised Sujatha & GS \\
Sujatha & S \\
Aradhana & A \\
Lindley & L \\
Exponential & E \\
Weibull & W \\
\hline
\end{tabular}

Table 7: MLEs, AIC, CAIC, and BIC statistics of the fitted distributions of dataset

\begin{tabular}{lcccc}
\hline Distribution & Parameters & AIC & CAIC & BIC \\
\hline GIW & $2.1159,2.2149,5.0579$ & 36.7857 & 38.2857 & 39.7729 \\
OFIW & $0.208,25.815,13.215$ & 44.253 & 45.753 & 42.156 \\
NMW & $0.1215,2.7837,8.227 \times 10-5,0.0003,2.7871$ & 51.173 & 55.459 & 47.678 \\
GS & $1.5712,222.235$ & 49.96 & 50.67 & 51.96 \\
S & 1.1367 & 59.49 & 59.71 & 60.49 \\
A & 1.1232 & 58.37 & 58.59 & 59.36 \\
L & 0.8161 & 62.49 & 62.71 & 63.49 \\
E & 0.5263 & 67.67 & 67.89 & 68.67 \\
W & $2.7870,2.1300$ & 45.1728 & 47.1643 & 45.8787 \\
\hline
\end{tabular}

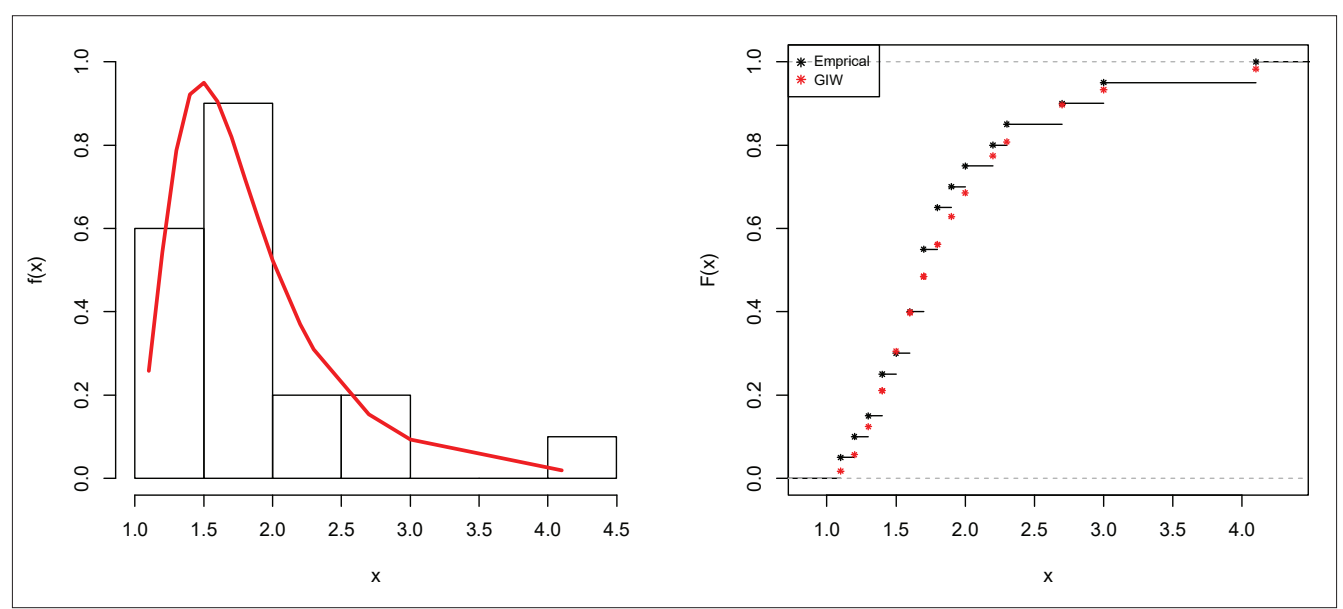

Figure 7: The histogram, empirical cdf and the cdf of GIW distribution for relief times data 
The second dataset consists of the relief times of twenty patients receiving an analgesic. The dataset in Table 5 is from Gross and Clark (1975). This dataset is studied and fitted with different models by Fayomi (2019). The values of the data set is provided in Table 5. The fitted distributions and the abbreviations for this dataset is listed in Table 6. Table 7 presents the lowest values of the AIC, CAIC, and BIC for the proposed model, which clearly indicates that the GIW distribution provides better fits than other models. The visual presentation also provides that the GIW distribution is adequately explaining the dataset.

\section{CONCLUSION}

In this article, we derived a new generalisation of inverse Weibull distribution using gamma class of distributions. Numerous mathematical characteristics for the proposed model was derived and discussed.

Some special cases of the proposed distribution were presented. Estimation of the model parameters were made through method of maximum likelihood. The evaluation of the model parameters were justified through simulation study. Two real dataset examples from the field of biomedical sciences were studied to check the applicability of the proposed model in real life phenomena. At the end, some remarks were stated.

\section{REFERENCES}

Abbas S., Taqi S.A., Mustafa F., Murtaza M. \& Shahbaz M.Q. (2017). Topp Leone inverse Weibull distribution: theory and application. European Journal of Pure and Applied Mathematics 10(1): 1005-1022.

Aleem M. \& Pasha G.R. (2003). Ratio, product and single moments of order statistics form inverse Weibull distribution. Journal of Statistics 10(1): 7-8.

Benkhelifa L. (2017). Log-Lindley generated family of distributions, arXiv:1705.03695v1, submitted on 10 May 2017.

Bjerkedal T. (1960). Acquisition of resistance in guinea pigs infected with different doses of virulent tubercle bacilli. American Journal of Hygiene 72(1): 130-148.

DOI: https://doi.org/10.1093/oxfordjournals.aje.a120129

Brito C.C., Gomes-Silva F., Rłgo L.C. \& de Oliveir W.
Rosa (2017). A new class of gamma distribution. Acta Scientiarum 39(1): 79-87.

DOI: https://doi.org/10.4025/actascitechnol.v39i1.29890

Calabria R. \& Pulcini G. (1990). On the maximum likelihood and least-squares estimation in the inverse Weibull distributions. Statistica Applicata 2(1): 53-66.

Fayomi A. (2019). The odd Frechet inverse Weibull distribution with application. Journal of Nonlinear Sciences and Applications 12: 165-172.

DOI: https://doi.org/10.22436/jnsa.012.03.04

de Gusmao F.R.S., Ortega E.M.M. \& Cordeiro G.M. (2009). The generalized inverse Weibull distribution. Statistical Papers 3: 362-371.

Gross A.J. \& Clark V. (1975). Survival Distributions: Reliability Applications in the Biomedical Sciences. John Wiley and Sons, USA.

Hanook et al. 2009 (Hanook, S., Shahbaz, M.Q., Mohsin, M. and Golam Kibria, B.M., 2013. A note on beta InverseWeibull Distribution. Communications in Statistics-Theory and Methods, 42(2), pp.320-335.)

Keller A.Z. \& Kanath A.R.R. (1982). Alternative reliability models for mechanical systems. Third International Conference on Reliability and Maintainability. Toulouse, France, pp. 411-415.

Lazhar Benkhelifa (2017). Log-Lindley generated family of distributions, arXiv:1705.03695v1, Submitted on 10 May 2017.

Mahdavi A. \& Jabbari L. (2017). An extended weighted exponential distribution. Journal of Modern Applied Statistical Methods 16(1): 296-307. DOI: https://doi.org/10.22237/jmasm/1493597760

Mahmoud M.A.W., Sultan K.S. \& Amer S.M. (2003). Order statistics from inverse Weibull distribution and characterizations. METRON LXI.3: 389-401.

Nelson W. (1982). Applied Life Data Analysis. John Wiley and Sons, USA.

DOI: https://doi.org/10.1002/0471725234

Shahbaz M.Q., Saman S. \& Butt N.S. (2012). The Kumaraswamy inverse Weibull distribution. Pakistan Journal of Statistics and Operation Research 3: 479-489.

DOI: https://doi.org/10.18187/pjsor.v8i3.520

Shanker R., Fesshayeand H. \& Selvaraj S. (2015). On modeling of lifetimes data using exponential and Lindley distributions. Biometrics and Biostatistics International Journal 2(5): 140-147. DOI: https://doi.org/10.15406/bbij.2015.02.00042

Shuaib M.K. \& King R. (2016). New generalized inverse Weibull distribution for lifetime modeling. Communications for Statistical Applications and Methods 3(2): 147-161. DOI: https://doi.org/10.5351/CSAM.2016.23.2.147 